\title{
Correction to: Transplantation of Human Chorion-Derived Cholinergic Progenitor Cells: a Novel Treatment for Neurological Disorders
}

\author{
Alireza Mohammadi ${ }^{1}$ - Ali Maleki-Jamshid ${ }^{2,3,4}$ - Davood Sanooghi ${ }^{5} \cdot$ Peiman Brouki Milan $^{3,6} \cdot$ Arash Rahmani $^{3,7}$. \\ Farshid Sefat $^{8} \cdot$ Koorosh Shahpasand $^{9} \cdot$ Mansoureh Soleimani $^{2,3} \cdot$ Mehrdad Bakhtiari $^{2,3} \cdot$ Rafie Belali $^{10}$. \\ Faezeh Faghihi ${ }^{3} \cdot$ Mohammad Taghi Joghataei $^{2,3,4} \cdot$ George Perry $^{11}$ - Masoud Mozafari ${ }^{3,6,12}$
}

Published online: 26 April 2018

(C) Springer Science+Business Media, LLC, part of Springer Nature 2018

\author{
Correction to: Mol Neurobiol \\ https://doi.org/10.1007/s12035-018-0968-1
}

The original version of this article unfortunately contained a mistake in the affiliation. Affiliation 1 should be read as "Neuroscience Research Center, Baqiyatallah University of Medical Sciences, Tehran, Iran". The original article has been corrected.

Mistakes were noted also at the figure captions as the descriptions of Figs. 3, 4, and 5 were interchanged. The authors hereby publish the correct captions below.

Fig. 3 Transplanted of BFCN progenitor cells migrate into the adult hippocampus and express markers of

The online version of the original article can be found at https://doi.org/ 10.1007/s12035-018-0968-1

Faezeh Faghihi

faghihi.f@iums.ac.ir

$\triangle$ Mohammad Taghi Joghataei

mt.joghataei@yahoo.com

$\checkmark$ George Perry

george.perry@utsa.edu

Masoud Mozafari

mozafari.masoud@gmail.com

1 Neuroscience Research Center, Baqiyatallah University of Medical Sciences, Tehran, Iran

2 Department of Anatomy, School of Medicine, Iran University of Medical Sciences, Tehran, Iran

3 Cellular and Molecular Research Center, Iran University of Medical Sciences, Tehran, Iran

4 Department of Neuroscience, School of Advanced Technologies in Medicine, Iran University of Medical Sciences, Tehran, Iran

5 Department of Genetics, Faculty of Biological Sciences, Shahid Beheshti University, Tehran, Iran cholinergic neurons. a Serial sections of $500 \mu \mathrm{m}$ of the hippocampus (nuclei and grafted BFCN progenitor cells were stained with DAPI (blue) and DiI (red), respectively) and a distribution of transplanted BFCN progenitor cells expressing DiI after 1 and 3 months were determined by IHC which is showed that transplanted BFCN progenitor cells dispersed after grafting into recipient rats in spite of the wide-ranging hippocampal cell death resulting from $A \beta$ injection. Scale bar $=200 \mu \mathrm{m}$

Fig. 4 Immunohistochemical images of rat brain after 1 and 3 months cell transplantation. Red DiI-labeled cells were detected in injured sites in hippocampus. The total number of the NEUN, ChAT, ISL, and nestin-positive

6 Department of Tissue Engineering \& Regenerative Medicine, Faculty of Advanced Technologies Medicine, Iran University of Medical Sciences (IUMS), Tehran, Iran

7 Mental Health Research Center, Tehran Institute of PsychiatrySchool of Behavioral and Sciences Mental Health, Iran University of Medical Sciences, Tehran, Iran

8 School of Engineering, Design and Technology-Medical Engineering, University of Bradford, Bradford, West Yorkshire, UK

9 Department of Stem Cells and Developmental Biology, Cell Science Research Center, Royan Institute for Stem Cell Biology and Technology, ACECR, Tehran, Iran

10 Physiology Research Center and Department of Physiology, Faculty of Medicine, Iran University of Medical Sciences, Tehran, Iran

11 Neurosciences Institute and Department of Biology, College of Sciences, University of Texas at San Antonio, San Antonio, TX, USA

12 Bioengineering Research Group, Nanotechnology and Advanced Materials Department, Materials and Energy Research Center (MERC), PO Box 14155-4777, Tehran, Iran 
cells were counted in each field and data showed that BFCN efficiently engrafted in hippocampus compared with hCMSCs posttransplantation. The majority of transplanted BFCN progenitor cells significantly express nestin on 1 month and NEUN and ChAT on 3 months nuclei were stained with DAPI. Scale bar = $100 \mu \mathrm{m}$

Fig. 5 Effects of BFCN progenitor cell transplantation on beta amyloid plaques formation. a Beta amyloid was detected in rat hippocampus (red). GFAP was identified in activated astrocytes (green). b There were no significant differences in total number of beta amyloid plaques between groups before and after treatment. c The concentration of secreted Ach and activity of AchE were quantified by ELISA analyses for hCMSC-derived BFCN on 3 months after transplantation $(* * * P<0.001, * P<0.05)$. Scale bar $=100 \mu \mathrm{m}$ 